\title{
Detecting Data Integrity Failures
}

\author{
W. List CA FBCS \\ The Kingswell Partnership \\ 46 Snakes Lane \\ Woodford Green \\ Essex IG8 ODF \\ $U K$ \\ Telephone/Fax: +441815046480 \\ Email:100416.13@compuserve.com
}

\begin{abstract}
All data in systems will contain integrity failures (errors). Their causes are legion and endemic. Their effect is random and sometimes disastrous. This paper sets out a basic theory of how these errors can be managed in a secure system. In summary the theory requires that processing and reporting programs contain error detection functionality and that formal procedures exist for (senior) users to be seen to be responsible for the ongoing correctness of data.
\end{abstract}

Keywords
Error, Integrity, Detection, Data, Users, Information

1 INTRODUCTION

Today, it is still the most common excuse for errors in invoices etc. that the computer malfunctioned or it can not be done on the system. Everyone has heard of the utility bill for zero which has to be paid, or the invoice for far too much, or the real phantom withdrawals from an ATM. The stories are legion and create a feeling of unease amongst users and the public.

All programs contain 'bugs' (except for the very smallest that have been very well tested); fortunately most of the 'bugs' remain latent and do not upset the processing.

All people make mistakes; most are fortunately of little consequence. 
The purpose of this paper is to explore the requirement to find the errors in the results of processing before they cause further problems. It is divided into the following sections:

- Assumptions.

- What is an error?

- What data is involved?

- How to find errors.

- When should errors be found?

- Risk analysis.

In the paper Integrity in Information Systems $\{1\}$ it was postulated that Integrity should be defined as "sufficiently right at the time of use for the purpose to which the user wishes to put the output". In that paper the impact of data or reporting error on the user's perception of Integrity was identified as a material element which would affect the user's decision on "fit for purpose".

In the Preliminary Draft of the Common Criteria for Information Technology Security Evaluation $\{2\}$ Integrity is defined as "The property that assumptions about the known or expected state of information or resources remain true". Clearly the information user's assumptions about the level of error in the information being used have a material bearing on his/her perception of an expected state of the information received from the computer. Version 1 of the Common Criteria includes a functionality component which addresses the detection of Integrity failures in the stored user data.

This paper is set against a background of future systems where :

- users access a worldwide organisation network and extract the data and information they require;

- users access many other worldwide networks to obtain information as required;

- users create their own reports from the information obtained;

- business processes are substantially automated, integrated and use the worldwide networks to transfer data and information between and within organisations;

- there are a limited number of people involved in administration; and

- there is substantial pressure for up to the minute availability of information.

The examples in this paper will be framed in the context of a system containing the record of the events within an organisation held in a worldwide distributed logical database.

\subsection{User assumptions}

The user is expected to assume that the information received will only contain insignificant errors. This assumption is expected to remain true even if the user 
codes their own reporting programs and may not fully understand the data descriptions held in the data dictionary.

\subsection{Program assumptions}

Programs accessing data usually assume that it correct. Unless there are tests within the program to detect errors then the processing will continue on the assumption there are none. Clearly if processing fails (interrupts) due to data error then the fact that an error exists is obvious.

\section{WHAT IS AN ERROR?}

An error is any representation of real world events, either individually or in total, which fails to reflect the entirety of the event(s) accurately and in many cases also in a timely manner. This definition is constrained because, usually, not all elements of real world events are recorded and an error within the system can only be construed against the data that is expected to be there.

Users may expect other things to be recorded and consider the representation to be wrong if they are not. This is a matter addressed in the business requirement specification and not discussed further in this paper.

Errors arise from many causes, including:

- mistakes in the original input;

- failure to correctly input the data;

- failure to process the data by application programs in accordance with the specifications (including errors which do not cause a program failure);

- failure of the application software specifications to fully reflect the business requirement;

- failure of the application software interaction with the other software/hardware being used in processing;

- failure to recover completely or correctly after an incident;

- failure to specify output requirements correctly; and

- failure to understand, or interpret correctly, the resulting output.

The data in any system may be categorised as:

Parameters: - data which govern the operation of application or system software, including IT security software.

Standing Data: - data relating to specific activities which is subject to infrequent alteration. 
Tables: - data relating to specific activities which is held for varying lengths of time. (This data is not to be confused with data held in tables as part of a database structure).

Transactions: - the unit of entry or creation of data which represents the whole or part of a single real world event.

The extent of damage that may be caused by an error in the data increases with the length of time that data is held for processing and by the number of transactions that may be effected. In principle therefore it is more important to ensure no errors exist in parameters, standing data and tables than in individual transactions.

\section{HOW TO FIND ERRORS}

There are two basic ways in which errors can be detected:

- by manual scrutiny of data; and

- by programmed examination of data.

\subsection{Manual scrutiny}

In well run systems there will be a procedure for regular management scrutiny of (some or all) of the details of parameters, standing data and tables. This procedure will display the current values of the data and the responsible manager will be expected to check this and confirm its correctness. This procedure involves management in the day to day security of the system.

Management and clerical staff also use the results of processing in their day to day activities. They also create their own extracts of data for use. Most users, most of the time, have a perception of what the results should be and therefore can judge if the results provided are within an acceptable tolerance or not. Where results are not within the acceptable tolerance then they will make enquiries to find out why.

The unreliability of this necessary scrutiny activity arises because:

- where errors exist in multiple reports there is a danger that either two (or even more) people initiate necessary corrective action or no one does because they think others will do so; and

- when the information conforms to user perceptions but is in fact materially wrong.

This process is therefore not an effective or reliable substitute for a thorough check from time to time.

\subsection{Programmed examination}

There are three types of test that can be performed. These are: 
- identification of data field contents which are either impossible (e.g. invalid dates, etc. ) or not permitted by the application (e.g. sex is $\mathrm{N}$ - not M or F, etc. ). This class of test should be set up at the time of writing the application and cover all possibilities particularly those data items not normally available to users (e.g. delete flags, etc. );

- identification of (potentially) erroneous data. This class of test seeks identify errors in records which conform to the application rules but which are (potentially) illogical or wrong from a business perspective (e.g. a mortgage rate of $1 \%$, miscalculation of taxes, duplicate customers or suppliers, missing addresses, etc.). This class of test may also be directed at specific anti-fraud checking; and

- recomputation of totals or created data.

In order to recompute data it is necessary to have available the expected result in order to compare the checking result with it. Where the totals are of all data (or some elements within all records) the application must create the totals for comparison - a rare event these days except for totals required in financial (general ledger) accounting.

To reprogram very complex calculations including myriad minor variations is likely to prove uneconomic unless it is essential that the computations are always accurate (e.g. in a navigation system). A standard approximation is sufficient provided that 'errors' are only reported outside a tolerance.

6 WHEN SHOULD ERRORS BE FOUND?

\subsection{Principles}

The latest time for detecting errors is before any erroneous data is used in circumstances where it would cause loss, inconvenience or embarrassment to the organisation.

Clearly if errors are to be detected, the sooner this is done in processing the better, because it reduces the risk of accidental use of erroneous data.

\subsection{Detect errors on input}

In batch systems all input can be subjected to extensive validation, however in modern systems the trade off between validation, data structures and response times has led to input being subjected to far less stringent validation. Given that in modern systems it is not possible to control the order in which data is input, the usual extent to which new input can be validated is:

- to ensure that it is capable of being processed (e.g. numerics where they ought to be, codes as permitted by the application, etc. ); and

- to ensure that it is not in conflict with other relevant data present at the time of input. 


\subsection{Detection of errors on output or processing}

Other more extensive tests should be performed by the formal reporting programs, or other formal processing programs, so that anomalies in the proposed or potential output are found and reported for action. This is simply because formal reporting or processing programs usually process all the relevant data and can therefore perform the checks as a by-product of their processing. To achieve these checks requires disciplined programming where no assumptions are made as to the correctness of data being used.

Such tests are particularly important in programs which create commitments for the organisation (e.g. contracts, orders, payroll, etc. ) and where reporting is to external authorities (e.g. taxation returns, etc. ).

Clearly there will require to be policy decisions on the action to take when an anomaly is detected:

- is it ignored? or

- is it included as if not in error in the processing and later any required correction made? - and usually a limit set to terminate execution if too many anomalies are found.

\subsection{Formal error detection programs}

In addition to the checking during input, processing and output there is a requirement for formal checking programs to be run simply to detect residual errors. Such programs should not only be applied against current data but also against the archive. Such programs should not assume that any checks have been done previously and repeat all possible checks on the data.

\section{$7 \quad$ RISK ANALYSIS}

In security terms it is unacceptable never to formally search for data integrity failures therefore the risk analysis techniques should be directed at determining the frequency of checking and not whether it should be done at all or only to some data elements.

It is important to remember the following when determining the frequency of error detection:

- if there is an error and the data is not used it does not matter;

- finding errors costs processing time (and possibly causes delay in response time). The processing cost needs to be judged against both the people cost of resolving any problems occasioned by using erroneous data and the marginal cost of surplus capacity in any system (for example overnight or weekends or even the standby hot site); 
- it is more important to find errors in data which governs the processing of many transactions than it is to find errors in single transactions; and

- small errors cause considerable inconvenience to staff (or the public) using the data and, if there are a significant number of these reinforce the belief that systems do not work.

\section{CONCLUSION}

Data will always contain errors; the majority of them are insignificant. The volume of errors in the data need to be managed so that the risk of erroneous management decisions or business commitments is maintained at an acceptable level.

The use of risk analysis, in this context, is to determine the timing and extent of the checking of the stored data to detect errors. It is cost effective to include checks within reporting and processing programs so that errors are reported as a byproduct of other necessary work and because any resulting errors from the process are trapped at the point in time when action can be taken to avoid damage to the organisation.

Procedures to detect integrity failures need to be very closely linked to (end) user correction procedures. How best to make a correction to a detected error is a decision which can often only be taken once the error has been identified.

The failure to implement effective error detection is now and will continue to be the main cause of loss of confidence in systems. If the promise of world wide electronic trading and information services is to be realised the systems must include error detection. How best to implement this is a substantial challenge to IT security specialists today.

9

\section{REFERENCES}

List, W. and Melville, W.R. (1994) Integrity in Information Systems, City University Business School Working Paper

Preliminary draft of the Common Criteria for Information Technology Security Evaluation Version 0.9 and version 1.0 


\section{BIOGRAPHY}

\section{William List CA FBCS}

He is a director of The Kingswell Partnership; a consultancy specialising in all aspects of business risk limitation. He served for over 15 years as a computer auditor partner in KPMG in UK. He is an acknowledged international expert in the use of control and security techniques in application systems, including those involving networks, EDI and distributed processing.

He is currently:

Chairman of the British Computer Society (BCS) Security Committee Visiting Fellow City University Business School BCS representative on IFIP TC 11 - Information Security Technical Committee Member of the Institute of Chartered Accountants of Scotland IT Committee Member of the Electronic Commerce Association accounting special interest group 